Abanico Agroforestal. Enero-Diciembre 2020; 2:1-11. http://dx.doi.org/10.37114/abaagrof/2020.2

Artículo Original. Recibido: 16/06/2019. Aceptado: 15/01/2020. Publicado: 15/02/2020.

\title{
Determinación de minerales en hígado y en sangre de ovejas alimentadas con altas dosis de pollinaza por periodos prolongados
}

Determination of minerals in liver and blood of sheep fed with high poultry manure doses for prolonged periods

\section{Peña-Parra Bladimir ${ }^{1}$, Duran-Puga Noe ${ }^{2}$, Alejo-Santiago Gelacio ${ }^{3}$, Escalera- Valente Francisco ${ }^{1}$, Herrera-Corredor Alejandra ${ }^{4}$, Rivas-Jacobo Marco ${ }^{4}(\mathbb{D}$, Martínez-González Sergio' ${ }^{1}$ (D) *Ávila-Ramos Fidel ${ }^{5}$}

${ }^{1}$ Universidad Autónoma de Nayarit, Unidad Académica de Medicina Veterinaria y Zootecnia. Nayarit, México. ${ }^{2}$ Centro Universitario de Ciencias Biológicas y Agropecuarias. Universidad de Guadalajara. México. 3Universidad Autónoma de Nayarit, Unidad Académica de Agricultura. Nayarit, México. 4Universidad Autónoma de San Luis Potosí, Facultad de Agronomía y Medicina Veterinaria. San Luis Potosí, México. 5Universidad de Guanajuato, Medicina Veterinaria y Zootecnia. Guanajuato. México. *Autor de correspondencia: Universidad de Guanajuato, División de Ciencias de la Vida, Medicina Veterinaria y Zootecnia. Ex Hacienda El Copal, km. 9 Carretera Irapuato-Silao, CP. 36500, Irapuato, Guanajuato. México. bladiuan73@gmail.com, noeduranpuga@yahoo.com.mx, gelacioalejo@hotmail.com, franes08@hotmail.com, alejandra.herrera@uaslp.mx, marco.rivas@uaslp.mx, sergiotepic@hotmail.com, ledif@hotmail.com.

\section{RESUMEN}

El objetivo fue medir los niveles de minerales en hígado y en sangre de ovinos alimentados con altos niveles de pollinaza. Se consideraron dos grupos experimentales, un grupo tratado (vientres ovinos que consumieron pollinaza por al menos tres años) y un grupo testigo (corderos en engorda que no consumieron pollinaza). Se sacrificaron al azar seis animales de cada grupo $(n=6)$, previo al sacrificio se colectaron las muestras sanguíneas, y posteriormente se tomaron muestras de tejido hepático. Los minerales analizados fueron cobre $(\mathrm{Cu})$, hierro $(\mathrm{Fe})$ y zinc $(\mathrm{Zn})$. En el grupo tratado, las concentraciones en hígado de $\mathrm{Cu}$, Fe y $\mathrm{Zn}$ fueron de 176.23, 9.58, y $72.63 \mathrm{mg} / \mathrm{L}$, respectivamente; mientras que en el grupo testigo fueron de $85.35,13.41$ y $112.0 \mathrm{mg} / \mathrm{L}$, respectivamente. Las determinaciones de Cu del grupo tratado fueron superiores tanto en hígado como sangre $(p<0.05)$. Sin embargo, a pesar del tiempo de exposición al consumo de pollinaza, los animales no presentaron signos de intoxicación por cobre, lo que pudo estar condicionado por la baja concentración del mineral en las excretas avícolas.

Palabras clave: ovinos, minerales salud, cobre, hierro, zinc y pollinaza.

\begin{abstract}
The objective was to measure the levels of minerals in the liver and blood of sheep, fed with high levels of poultry manure. Two experimental groups were considered: a treated group (ovine bellies that consumed poultry manure for at least three years) and a control group (lambs in fattening that did not consume poultry manure). Six animals $(n=6)$ from each group were randomly sacrificed, blood samples were collected prior to slaughter, and hepatic tissue samples were subsequently taken. The minerals analyzed were copper $(\mathrm{Cu})$, iron (Fe) and zinc ( $\mathrm{Zn})$. In the treated group, concentrations of $\mathrm{Cu}$, Fe and $\mathrm{Zn}$ were 176.23, 9.58, and $72.63 \mathrm{mg} / \mathrm{L}$, respectively. While in the treated group they were $85.35,13.41$ and $112.0 \mathrm{mg} / \mathrm{L}$, respectively. The $\mathrm{Cu}$ determinations of the treated group were higher in both liver and blood $(p<0.05)$. However, in spite
\end{abstract}


of the time of exposure to poultry manure consumption, the animals showed no signs of copper intoxication, which could be conditioned by the low concentration of the mineral in the poultry excreta.

Keywords: ovines, health, copper, iron, zinc, poultry manure.

\section{INTRODUCCIÓN}

En México, la producción de ovinos se ve limitada por los altos costos de los alimentos; además la ovinocultura aún es de traspatio; es decir, como actividad económica secundaria (Martínez et al., 2011). Este tipo de explotación da lugar a la utilización de desechos industriales, tal como la pollinaza en la alimentación animal. México está consolidado como el quinto productor de pollo y huevo a nivel mundial (SAGARPA, 2016), por lo que la producción de pollinaza es muy alta, lo que la convierte en un residuo potencial para la alimentación ovina.

El uso de la pollinaza, como suplemento alimenticio, se debe a la facilidad de adquisición y costo moderado. Entre sus ventajas es el aporte importante de proteína y de minerales para los animales, sobre todo de fósforo disponible. Entre las desventajas se encuentra su elevado contenido de cobre, mismo que puede provocar intoxicación (Castellanos, 2007). Existen recomendaciones de no utilizar la pollinaza por tiempos prolongados ni en altas concentraciones (Ríos et al., 2005), para evitar la presentación de intoxicaciones; sin embargo, el productor abusa del empleo de la pollinaza, incluyendo altos niveles en la dieta; pensando en alimentar mejor y más barato a su ganado, lo que resulta contraproducente Castellanos, 2007).

El cobre $(\mathrm{Cu})$ es un elemento traza esencial para la mayoría de los procesos biológicos de plantas y animales. Es útil para el metabolismo normal de hierro, síntesis de elastina y colágeno, producción de melanina y para la integridad del sistema nervioso central (Kimberling, 1998); también es un cofactor de muchas cuproenzimas, pero es extremadamente tóxico en exceso (Horn and Tumer, 1999; Mercer, 2001).

Todos los organismos vivos han desarrollado mecanismos homeostáticos altamente especializados para reclutar, trasladar y eliminar el cobre; así como para neutralizar su efecto tóxico (Dameron and Harrison, 1998; Harris, 2000; Mercer, 2001). Varias especies de animales muestran variación en su tolerancia a los incrementos de los niveles de cobre en la dieta (Howell y Gooneratne, 1987).

Sin duda, los ovinos son los más susceptibles a la toxicidad crónica de cobre; sin embargo, en cuanto a la resistencia a la toxicidad por cobre, existen diferencias entre razas, pues algunos autores comentan que la toxicidad por cobre, ocurre más frecuentemente en la raza Dorper que en la Merino (Bath, 1979; Harrison et al., 1987). 
Por otro lado, está la cuestión de la inocuidad de los alimentos, algunos autores (Ríos et al., 2005) comentan la importancia de la salud animal, sobre todo cuando se evalúan la cama de pollo y la gallinaza, como suplemento en la alimentación animal; pues los productos que se generan a partir de esos animales serán consumidos por el hombre. Por lo tanto, el objetivo del presente trabajo fue determinar las concentraciones de cobre, hierro y zinc en hígado y sangre; así como valorar la salud de los animales en una granja que suplementaba a sus ovejas con alto contenido de pollinaza en la dieta.

\section{MATERIAL Y MÉTODOS}

Para la realización de este trabajo y del cuidado de los animales, se tomaron en cuenta los lineamientos de la Norma Oficial Mexicana (NOM-062-ZOO-1995): Especificaciones técnicas para el cuidado y uso de animales de laboratorio, explotaciones ganaderas, granjas, centros de producción, reproducción y cría.

El trabajo se realizó en la granja "El Refugio" la cual se encuentra localizada en Lo de García, Tepic, Nayarit. La región cuenta con clima cálido sub húmedo, lluvias en verano, temperatura promedio anual de $21.3^{\circ} \mathrm{C}$, precipitación promedio de $1152.3 \mathrm{~mm}$ y una altitud de $915 \mathrm{~m}$ sobre el nivel del mar (Ríos et al., 2005). Se contaba con 200 vientres ovinos (grupo tratado, suplementados con pollinaza), y 30 corderos en engorda (grupo testigo, alimentados sin pollinaza).

La pollinaza fue adquirida en una empresa avícola de la región, a dicha pollinaza se le realizó análisis de los minerales de $\mathrm{Cu}, \mathrm{Fe}$ y $\mathrm{Zn}$. El manejo de las excretas se realizó siguiendo las indicaciones de la Norma Oficial Mexicana (NOM-044-ZOO-1995).

\section{Grupos experimentales}

Grupo tratado: la raza de estas hembras era Pelibuey/Katahdin; las edades oscilaban entre los 10 meses y los 5 años. La condición corporal se situaba entre 2 y 3.5 siguiendo la clasificación de De Lucas (2007). El manejo de los vientres (durante los últimos tres años) era en un sistema de alimentación semiestabulado, con pastoreo y acceso ad libitum al suplemento. Dicho suplemento contenía el $60 \%$ pollinaza, $10 \%$ de grano de maíz, $28 \%$ de melaza y $2 \%$ de mezcla comercial de minerales para ovinos. Cada kilogramo de esta mezcla mineral contenía: calcio (Ca) $130 \mathrm{~g}$, fósforo (P) $50 \mathrm{~g}$, sodio $(\mathrm{Na})$ 109 g, Cloro (Cl) 200 g, Hierro (Fe) 4.3 g, Magnesio (Mg) 10 g, Manganeso (Mn) $3.3 \mathrm{~g}$, minerales traza y el resto salvado de trigo, melaza de caña y saborizante vegetal.

Grupo testigo: la edad promedio de estos animales era de seis meses, de raza Pelibuey/Katahdin. En estos animales el sistema de producción era totalmente estabulada; de esta manera, la alimentación fue de forma continua y consumían una 
dieta comercial a base de maíz grano, rastrojo de maíz, sorgo grano, soya, melaza, canola y $2 \%$ de la mezcla comercial de minerales para ovinos ya descrita.

\section{Sacrificio de animales}

Se sacrificaron 6 animales (elegidos al azar) de cada grupo; aunque en el grupo tratado se procuró que los animales tuvieran más de cuatro años de edad, con la finalidad de asegurar que habían consumido pollinaza durante tres años.

Para llevar a cabo este trabajo y el cuidado de los animales, se tomaron en cuenta las pautas de la Norma Oficial Mexicana (NOM-062-ZOO-1995): Especificaciones técnicas para el cuidado y uso de animales de laboratorio, granjas ganaderas y granjas, centros de producción, reproducción y cría. Los animales fueron sacrificados siguiendo las recomendaciones de la Norma Oficial Mexicana (NOM-033-ZOO-1995): sacrificio humanitario de animales domésticos y salvajes.

La toma de muestras sanguíneas se realizó antes del sacrificio de los animales, mediante venopunción yugular, con aguja vacutainer de 21G; la sangre obtenida se colocó en tubos heparinizados de $3 \mathrm{cc}$. Una vez muertos los animales, se tomaron muestras de tejido hepático del lóbulo izquierdo. Se determinaron las concentraciones de $\mathrm{Cu}, \mathrm{Fe} \mathrm{y} \mathrm{Zn;}$ en sangre, hígado y pollinaza, mediante espectrofotometría de absorción atómica con ayuda de un equipo Spectra AA de Marca Varian.

Las muestras de sangre se analizaron con una dilución 1:1 (vol:vol), con agua destilada, e inmediatamente se procedió a realizar la lectura en el equipo de absorción atómica, con la lámpara respectiva para cada elemento. En el caso de muestras de hígado y de pollinaza se requirió de un proceso de digestión gradual en una plancha de digestión, iniciando en $50{ }^{\circ} \mathrm{C}$, hasta llegar a $250 \stackrel{\circ}{\circ}$, para la destrucción total del tejido. Una vez que terminó el proceso de digestión, se obtuvo una muestra transparente, y posteriormente se realizó una dilución de la muestra en matraces volumétricos de $25 \mathrm{ml}$, con agua destilada, y se procedió a la lectura de los elementos, en el equipo de absorción atómica. Los ácidos utilizados fueron, ácido nítrico con ácido perclórico y grado reactivo, en proporción 2:1 (vol:vol) (Alcántar y Sandoval, 1999). Las recomendaciones de MediCalc® (MediCalc®, 2017) se utilizaron para la conversión a unidades internacionales (de $\mu \mathrm{g} / \mathrm{dl} \mathrm{a} \mu \mathrm{mol} / \mathrm{L}$ ).

\section{Análisis estadístico}

Se realizó un análisis descriptivo que incluyó el valor medio, la desviación estándar, mínimo y máximo. El contraste de hipótesis se realizó mediante una prueba de t-Student para muestras independientes; estos análisis fueron realizados con el programa estadístico SPSS Versión 20.0 (IBM, 2011). 


\section{RESULTADOS Y DISCUSIÓN}

\section{Valores de pollinaza}

De las muestras de pollinaza analizadas, el hierro fue el que se encontró en mayor concentración, seguido del zinc y por último el cobre (tabla 1). Los valores de los minerales analizados siguen la misma tendencia de presentación que la descrita por Pacheco et al. (2003), es decir las concentraciones de hierro son las más altas, seguidas de las de zinc y por último el cobre.

Tabla 1. Niveles de minerales en pollinaza (ppm, base fresca)

\begin{tabular}{lcccc}
\hline & Promedio & $\begin{array}{c}\text { Desviación } \\
\text { estándar }\end{array}$ & Mínimo & Máximo \\
\hline Cobre $\mathrm{mg} / \mathrm{L}$ & 2.06 & 2.75 & 0 & 6.6 \\
Hierro $\mathrm{mg} / \mathrm{L}$ & 182.68 & 166.46 & 35.2 & 638.2 \\
Zinc $\mathrm{mg} / \mathrm{L}$ & 41.38 & 9.142 & 27.7 & 56.6 \\
\hline
\end{tabular}

Los valores de cobre en pollinaza (3.096 ppm) son inferiores a los publicados por Pacheco et al. (2003), quienes reportaron un promedio general de $82 \mathrm{ppm}$. Deshck et al. (1998); encontraron valores de cobre en pollinaza, en un rango de 29.0-122.7, con un promedio de 51.4; valores que son superiores a los encontrados en este estudio.

Es sabido que las dietas en ovinos deben contener cobre; sin embargo, existe controversia en cuanto a las concentraciones ideales. Tomando en cuenta las tablas de alimentación, éstas mencionan que los ovinos toleran hasta 25 ppm (NRC, 1985); Hartmans (1975) comentó que las dieta para ovinos con más de 15 ppm (15 mg/kg) de cobre, puede causar envenenamiento por este mineral; mientras que Plumlee (2004) comenta que la dosis tóxica comienza con 30 ppm, y como rangos normales menciona de 10-20 ppm. El nivel de cobre administrado en la presente investigación fue de 1.85 ppm (1.85 mg/kg), lo que está muy por debajo de los considerados como aconsejables.

Si bien es cierto que la concentración de cobre fue baja, se debe tener en cuenta tanto el alto porcentaje de inclusión de pollinaza en la dieta (60\%), así como el tiempo prolongado de exposición (tres años); lo que pudiera provocar la acumulación de cobre en hígado.

En cuanto al porcentaje de inclusión de pollinaza, existen trabajos en los que se ha incluido niveles desde un $50 \%$ (Pérez, 2004), $60 \%$ (Vivas, 2002) y hasta $85 \%$ (Mavimbela et al., 2000), con un periodo de exposición máximo de 4 meses.

Los resultados, en cuanto al tiempo de exposición al Cu necesario para producir intoxicación en ovinos, son diversos (Cantón et al., 1994), pues estos investigadores no 
registraron mortalidad dentro de los 91 días de alimentación con 87 ppm de Cu; mientras que otros grupos de trabajo describen mortalidad desde los 67 días, e incluso desde los 30 días de exposición al cobre, con niveles más bajos de este mineral en la dieta (67 ppm y 60 ppm, respectivamente) (Bostwick, 1982; Zervas et al., 1990).

\section{Valores en hígado}

Los resultados de las concentraciones de cobre, hierro y zinc, determinadas en hígado, se describen en la tabla 2. En esta tabla se observa que el cobre e hierro, fueron superiores en el grupo tratado; aunque sólo el cobre alcanzó diferencia estadística significativa $(p<0.05)$. Por otro lado, el zinc fue superior en el grupo testigo, aunque sin significación estadística.

El rango de valores de cobre hepático publicado por Underwood y Suttle (1999), es de 33.3-100 $\mu \mathrm{mol} / \mathrm{l}$, por lo que los valores de cobre hepático encontrado en el presente trabajo, en el grupo tratado y testigo, pueden considerarse como elevados (2819.2 y $1365.6 \mu \mathrm{mol} / \mathrm{l}$ respectivamente).

Tabla 2. Concentraciones medias de minerales en hígado de ovejas (ppm, base fresca) que consumieron pollinaza

\begin{tabular}{|c|c|c|c|c|c|c|}
\hline & & $\mathrm{N}$ & Promedio & $\begin{array}{l}\text { Desviación } \\
\text { estándar }\end{array}$ & Mínimo & Máximo \\
\hline \multirow{2}{*}{ Cobre mg/L } & $60 \%$ (Tratado) & 6 & $176.2^{\mathrm{a}}$ & 59.2 & 104.9 & 243.7 \\
\hline & $0 \%$ (Control) & 6 & $85.4^{\mathrm{b}}$ & 13.7 & 65.0 & 99.8 \\
\hline \multirow{2}{*}{ Hierro mg/L } & $60 \%$ (Tratado) & 6 & $72.6^{a}$ & 30.9 & 26.2 & 111.0 \\
\hline & $0 \%$ (Control) & 6 & $112.0^{\mathrm{a}}$ & 70.7 & 48.8 & 239.2 \\
\hline \multirow{2}{*}{ Zinc mg/L } & $60 \%$ (Tratado) & 6 & $9.6^{\mathrm{a}}$ & 3.5 & 3.1 & 13.1 \\
\hline & $0 \%$ (Control) & 6 & $13.4^{\mathrm{a}}$ & 2.9 & 8.5 & 16.1 \\
\hline
\end{tabular}

a,b Distintas literales indican diferencia estadística significativa entre los tratamientos $(p<0.05)$.

Plumlee (2004), comenta que en la mayoría de los rumiantes la acumulación de más de 250 ppm de cobre en hígado es considerada como tóxica; este valor es más alto del encontrado en hígado del grupo tratado (176.2 ppm), y casi el triple del valor del grupo testigo (85.3 ppm).

Los corderos muestreados por Sivertsen and Løverg (2014), en su primer año de estudio (noviembre, marzo y junio) promediaron un valor de $110 \mathrm{mg} / \mathrm{kg}$ en base húmeda; mientras que en el segundo año promediaron un total de $114 \mathrm{mg} / \mathrm{kg}$. Estos valores son superiores a los descritos en la presente investigación en corderos de 5 meses de edad $(85.35 \mathrm{mg} / \mathrm{kg})$; esta diferencia puede estar dada por el consumo de pastos que contenían niveles altos de cobre. 
Oruc et al. (2009) describieron valores de $302 \mathrm{mg} / \mathrm{kg}$ en base húmeda, estas muestras provenían de tres ovejas que habían muerto y que presentaban signos de intoxicación por cobre, estos valores son casi el doble de los de las borregas que consumieron pollinaza $(176.2 \mathrm{mg} / \mathrm{kg})$.

Otro factor que pudo contribuir es la resistencia de la raza, pues Harrison et al. (1987) comentaron que, en cuanto al metabolismo del cobre, existen diferencias genéticas entre las razas ovinas. En ese sentido Lewis et al. (1997) menciona que la raza Suffolk está particularmente en riesgo; mientras que Bath (1979) encontró que la toxicidad por cobre ocurre más frecuentemente en la raza Dorper, que en la raza Merino.

\section{Valores en sangre}

Los niveles de cobre, hierro y zinc, analizados en suero sanguíneo, fueron superiores en el grupo tratado con respecto al grupo testigo: aunque sólo el cobre mostró diferencias estadísticas significativas $(p<0.05)$ (tabla 3$)$.

Tabla 3. Valor medio de minerales en sangre de ovinos (ppm, base fresca) que consumieron

\begin{tabular}{lllcccc}
\hline & $\mathrm{N}$ & Promedio & $\begin{array}{c}\text { Desviación } \\
\text { estándar }\end{array}$ & Mínimo & Máximo \\
\hline \multirow{2}{*}{ Cobre $\mathrm{mg} / \mathrm{L}$} & $60 \%$ (Tratado) & 6 & $0.35^{\mathrm{a}}$ & 0.17 & 0.23 & 0.62 \\
& $0 \%$ (Control) & 6 & $0.12^{\mathrm{b}}$ & 0.03 & 0.06 & 0.14 \\
Hierro $\mathrm{mg} / \mathrm{L}$ & $60 \%$ (Tratado) & 6 & $36.25^{\mathrm{a}}$ & 30.91 & 8.90 & 95.20 \\
& $0 \%$ (Control) & 6 & $17.40^{\mathrm{a}}$ & 9.88 & 6.50 & 31.40 \\
\multirow{2}{*}{ Zinc $\mathrm{mg} / \mathrm{L}$} & $60 \%$ (Tratado) & 6 & $0.98^{\mathrm{a}}$ & 0.84 & 0.02 & 2.06 \\
& $0 \%$ (Control) & 6 & $0.93^{\mathrm{a}}$ & 0.36 & 0.40 & 1.37 \\
\hline
\end{tabular}

a, b Distintas literales indican diferencia estadística entre los tratamientos $(p<0.05)$.

Los resultados de ovejas que consumieron pollinaza, son ligeramente inferiores a los publicados por Mohammed et al. (2014), independientemente del estado fisiológico y de la estación; esto puede deberse a la raza (Bath, 1979; Lewis et al., 1997), aunque haría falta más estudios para apoyar la afirmación de que la raza Pelibuey es más resistente a la intoxicación por cobre.

\section{CONCLUSIÓN}

Los niveles de hierro y de zinc analizados en suero sanguíneo y en hígado del grupo tratado con respecto al grupo testigo, no mostraron diferencia estadística. Las determinaciones de cobre del grupo tratado fueron superiores, tanto en sangre como en hígado; sin embargo, ningún animal manifestó síntomas de intoxicación. 


\section{LITERATURA CITADA}

ALCÁNTAR GG, Sandoval VM. 1999. Manual de análisis químico de tejido vegetal. Guía de muestreo, preparación, análisis e interpretación. Publicación Especial 10. Sociedad Mexicana de la Ciencia del Suelo. Chapingo, Estado de México.

BATH GF.1979. Enzootic icterus-A form of chronic copper poisoning. Journal of the South Africa Veterinary Association. 50 (1): 3-14. https://www.ncbi.nlm.nih.gov/pubmed/551182 BOSTWICK JL. 1982. Copper toxicosis in sheep. Journal of the American Veterinary Medical Association. 180(4): 386-387. https://www.ncbi.nlm.nih.gov/pubmed/7037721

CANTÓN CJG, Moguel OY, Rojas RO, Sauri DR, Miranda SJ, Castellanos RAF. 1994. Estimación del daño inducido por el cobre de la pollinaza empleada en la alimentación de ovinos. Técnica Pecuaria en México. 32(2): 82-89.

http://cienciaspecuarias.inifap.gob.mx/index.php/Pecuarias/article/viewFile/3651/3071

CASTELLANOS RA. 2007. Condiciones que favorecen la intoxicación por cobre en ovinos alimentados con pollinaza. Fortalecimiento del Sistema Producto Ovino. Tecnologías para ovinocultores. Asociación Mexicana de Criadores de Ovinos (AMCO). Pp 19-20.

http://www.uno.org.mx/sistema/pdf/alimentacion/condicionesquefavorecenlaintoxicacion .pdf

DAMERON CT, Harrison MD. 1998. Mechanism for protection against copper toxicity. American Journal of Clinical Nutrition. 67: 1091S-1097S. https://www.ncbi.nlm.nih.gov/pubmed/9587158

DE LUCAS TJ. 2007. Evaluación de la condición corporal en ovejas. Fortalecimiento del Sistema Producto Ovinos. Tecnologías para ovinocultores. Asociación Mexicana de Criadores de Ovinos (AMCO). Pp. 135-140.

http://www.uno.org.mx/sistema/pdf/produccion/evaluaciondelacondicion.pdf

DESHCK A, Abo-Shehada M, Allonby E, Givens DI, Hill R. 1998. Assessment of the nutritive value for ruminants of poultry litter. Animal Feed Science and Technology. 73: 29-35. http://www.sciencedirect.com/science/article/pii/S0377840198001357

HARRIS ED. 2000. Cellular copper transport and metabolism. Annual Review of Nutrition. 20: 291-310. http://www.annualreviews.org/doi/abs/10.1146/annurev.nutr.20.1.291

HARRISON TJ, Van Ryssen JBJ, Barrowmen PR. 1987. The influence of breed and dietary molybdenum on the concentration of copper in tissues of sheep. South African Journal of Animal Science. 17: 104-110. http://www.sasas.co.za/influence-breed-anddietary-molybdenum-concentration-copper-tissues-sheep 
HARTMANS J. 1975. The frequency of occurrence of copper poisoning and the role of sheep concentrates in its merits enquiry. Tijdschrift voor Diergeneekunde 100: 379-382. In: Underwood EJ, Suttle NF. 1999. The Mineral Nutrition of Livestock. Third Edition. Wallingford: Ed. CABI Publishing. Pp. 283-342.

HORN N, Tumer Z. 1999. Molecular genetics of intracellular copper transport. Journal of Trace Elements in Experimental Medicine. 12: 297-313. http://onlinelibrary.wiley.com/doi/10.1002/(SICI)1520-670X(1999)12:4\%3C297::AIDJTRA3\%3E3.0.CO;2-E/abstract

HOWELL JM, Gooneratne SR. 1987. The pathology of the copper toxicity in animals. In: Howell JM, Gawthorne JM. (Eds), Copper in Animals and Man. Boca Raton, FL: C.R.C Press.

IBM CORP. Released. 2011. IBM SPSS Statistics for Windows, Version 20.0. Armonk, NY: IBM Corp.

INEGI. 2006. Anuario estadístico del estado de Nayarit. Instituto Nacional, Estadística Geografía e Informática Gobierno del Estado de Nayarit. México. Pp. 1-31. http://www.inafed.gob.mx/work/enciclopedia/EMM18nayarit/municipios/18017a.html

KIMBERLING CV. 1998. Jensen and Swift's disease of sheep. Third Edition. Philadelphia: Lea \& Febiger.

LEWIS NJ, Fallah-Rad AH, Connor ML. 1997. Copper toxicity in confinement-housed ram lambs. Canadian Veterinary Journal. 38: 496-498. https://www.ncbi.nlm.nih.gov/pmc/articles/PMC1576804/

MARTíNEZ GS, Macías CH, Moreno FLA, Zepeda GJ, Espinoza MME, Figueroa MR, Ruíz FM. 2011. Economic analysis of ovine production in Nayarit, México. Abanico Veterinario. $1(1): \quad 37-43 . \quad \mathrm{http}: / /$ new.medigraphic.com/cgibin/resumenl.cgi?IDARTICULO=45596

MAVIMBELA DT, Webb EC, Van Ryssen JBJ, Bosman MJC. 2000. Sensory characteristics of meat and composition of carcass fat from sheep fed diets containing various levels of broiler litter. South African Journal of Animal Science. 30(1): 26-32. https://www.ajol.info/index.php/sajas/article/view/3871

MEDICALC ${ }^{\circledR}$. 2017. http://www.scymed.com/es/smnxtb/tbcbglv1.htm Ultima consulta: 25 de marzo de 2017.

MERCER JFB. 2001. The molecular basis of cooper-transport diseases. Trends in Molecular Medicine. 7: 64-69. http://www.cell.com/trends/molecularmedicine/abstract/S1471-4914(01)01920-7 
MOHAMMED A, Campbell M, Yousse FG. 2014. Serum Copper and Haematological Values of Sheep of Different Physiological Stages in the Dry and Wet Seasons of Central Trinidad. Veterinary Medicine International. 2014: 972074. https://www.ncbi.nlm.nih.gov/pmc/articles/PMC4034437/

NORMA OFICIAL MEXICANA (NOM-033-ZOO-1995). Sacrificio humanitario de los animales domésticos y silvestres. Diario Oficial de la Federación, 7 de julio de 1995. https://www.gob.mx/senasica/documentos/normatividad-en-materia-de-salud-animal

NORMA OFICIAL MEXICANA (NOM-044-ZOO-1995). Campaña nacional contra la influenza aviar. Diario Oficial de la Federación, 25 de julio de 1996. https://www.gob.mx/senasica/documentos/normatividad-en-materia-de-salud-animal

NORMA OFICIAL MEXICANA (NOM-062-ZOO-1999). Especificaciones técnicas para la producción, cuidado y uso de los animales de laboratorio. Diario Oficial de la Federación, 18 de junio de 2001. https://www.gob.mx/senasica/documentos/normatividad-enmateria-de-salud-animal

NRC. National Research Council. 1985. Nutrient Requirements of Sheep. Sixth revised Edition. Washington, DC: National Academic Press. Pp. 99.

ORUC HH, Cengiz M, Beskaya A. 2009. Chronic copper toxicosis in sheep following the use of copper sulfate as a fungicide on fruit trees. Journal of Veterinary Diagnostic Investigation. 21(4): 540-543. https://www.ncbi.nlm.nih.gov/pubmed/19564507

PACHECO AJA, Rosciano GJL, Wilbert AVC, Alcocer VVM, Castellanos RAF. 2003. Cuantificación del contenido de cobre y otros minerales en pollinazas producidas en el estado de Yucatán. Técnica Pecuaria en México. 41 (2): 197-207. http://www.redalyc.org/articulo.oa?id=61341207

PÉREZ E. 2004. Efecto del consumo de gallinaza sobre la química sanguínea y alteraciones hepáticas en ovinos. Trabajo de Grado Ingeniero Agrónomo. Facultad de Agronomía, Universidad Central de Venezuela. Maracay, Venezuela. 51p. En: Ríos, AL, Combellas J, Alvarez ZR. 2005. Uso de excretas de aves en la alimentación de ovinos. Zootecnia Tropical. 23(2): 183-210.

PLUMLEE K. 2004. Clinical veterinary toxicology. First Edition. St. Louis, Missouri: Ed. Mosby. Pp. 504.

RIOS AL, Combellas J, Alvarez ZR. 2005. Uso de excretas de aves en la alimentación de ovinos. Zootecnia Tropical. 23(2): 183-210. http://www.bioline.org.br/request?zt05014 
SAGARPA. SECRETARÍA de Agricultura, Ganadería, Desarrollo Rural, Pesca y Alimentación. 2016. Gobierno Federal de México.

http://www.sagarpa.gob.mx/Delegaciones/nayarit/boletines/Paginas/BNSAGDIC272016 .aspx\# Última consulta: 12 de enero de 2017.

SIVERTSEN T, Løverg KE. 2014. Seasonal and individual variation in hepatic cooper concentrations in a flock of Norwegian Dala sheep. Small Ruminant Research. 116: 5765. http://www.sciencedirect.com/science/article/pii/S092144881300299X

UNDERWOOD EJ, Suttle NF. 1999. The Mineral Nutrition of Livestock. Third Edition. Wallingford: Ed. CABI Publishing. Pp. 283-342.

VIVAS L. 2002. Evaluación de la ganancia diaria de peso y posibles alteraciones en el tracto digestivo de ovinos alimentados con cama de pollo. Trabajo de Grado Ingeniero Agrónomo. Facultad de agronomía, Universidad Central de Venezuela. Maracay, Venezuela. Pp. 51.

https://www.researchgate.net/publication/48224840_Evaluacion_de_la_ganancia_diaria_de_pe so_y_de_posibles_alteraciones_en_el_tracto_digestivo_de_ovinos_alimentados_con_cama_de _pollos

ZERVAS G, Nikolaou E, Mantzios A. 1990. Comparative study chronic copper poisoning in lambs and young goats. Animal Production. 50: 497-506. https://www.cambridge.org/core/journals/animal-science/article/comparative-study-of-chroniccopper-poisoning-in-lambs-and-young-goats/98B84D4ECB306C348992C8B0062E7E91

Publica en las revistas Abanico. Publish in Journals Abanico. 